\title{
Pembrolizumab vorteilhaft bei PD-L1-positiven Patienten
}

Auch wenn in den letzten Jahren die Behandlung nichtkleinzelliger Bronchialkarzinome Fortschritte erzielen konnte, werden gerade bei Progress der Erkrankung weitere effektive Therapien dringend benötigt. Nun untersuchte die internationale Studiengruppe um R. S. Herbst die Wirksamkeit von Pembrolizumab bei PD-L1-positiven Tumoren.

Lancet 2016; 387: 1540 - 1550

In die offene Phase-II/III-Studie wurden Patienten mit vorbehandeltem nichtkleinzelligen Bronchialkarzinom eingeschlossen, die nach einer Therapie mit mindestens 2 Zyklen einer Platin-basierten Therapie oder Gabe geeigneter Tyrosinkinase-Inhibitoren bei entsprechenden Mutationen einen Progress zeigten. Voraussetzung für die Studienteilnahme war ferner die Expression von PD-L1 in mindestens $1 \%$ der Tumorzellen.

Die Teilnehmer wurden randomisert auf 3 Gruppen verteilt und erhielten alle 3 Wochen entweder Pembrolizumab in einer Dosierung von $2 \mathrm{mg} / \mathrm{kg}$ bzw. $10 \mathrm{mg} / \mathrm{kg}$
Körpergewicht oder Docetaxel in einer Dosierung von $75 \mathrm{mg} / \mathrm{m}^{2}$. Primäre Endpunkte waren das Gesamt- und das progressisonsfreie Überleben in der Gesamtkohorte sowie in einer Subgruppe von Patienten mit PD-L1-Expression in mindestens $50 \%$ der Tumorzellen. Zu den sekundären Endpunkten zählte u.a. die Sicherheit der Therapie.

In die Analyse gingen insgesamt $1034 \mathrm{~Pa}$ tienten ein, davon 345 in der Gruppe mit $2 \mathrm{mg} / \mathrm{kg}$ und $346 \mathrm{mit} 10 \mathrm{mg} / \mathrm{kg}$ Pembrolizumab und 343 in der Gruppe mit Docetaxel. Bis zum Studienende Ende September 2015 waren 521 Patienten verstorben. In der Studienpopulation betrug das mediane Gesamtüberleben unter $2 \mathrm{mg} / \mathrm{kg}$ Pembrolizumab 10,4 Monate, unter $10 \mathrm{mg} / \mathrm{kg}$ 12,7 Monate und unter Docetaxel 8,5 Monate. Das Gesamtüberleben war damit für Pembrolizumab in beiden Dosierungen deutlich länger als unter Docetaxel.

Für das progressionsfreie Überleben lauteten die entsprechenden Zahlen 3,9 ( $2 \mathrm{mg} / \mathrm{kg}), 4,0(10 \mathrm{mg} / \mathrm{kg})$ und 4,0 Monate (Docetaxel), wobei hier kein Unterschied zwischen den Gruppen bestand. Bei Patienten mit mindestens 50\% PD-L1-Expression waren unter beiden Dosierungen von Pembrolizumab sowohl Gesamt- als auch progressionsfreies Überleben länger als unter Docetaxel (14,9 und 17,3 Monate vs. 8,2 Monate bzw. 5,0 und 5,2 Monate vs. 4,1 Monate). Behandlungs-assoziierte Nebenwirkungen von Grad 3 bis 5 kamen unter Pembrolizumab seltener vor als unter Docetaxel (13 bzw. 16 vs. $35 \%$ ).

\section{Fazit}

Pembrolizumab verlängerte bei Patienten mit vorbehandeltem PD-L1-positivem, fortgeschrittenem nicht-kleinzelligem Bronchialkarzinom das Gesamtüberleben. Es sei eine neue, effektive Behandlungsoption mit einem günstigen Risikoprofil, so die Autoren.

Dr. Johannes Weiß, Bad Kissingen

des besten Arztes innerhalb der Gruppe vertrauen“, so Kurvers.

Für ihre Studie nutzten die Wissenschaftler 2 bereits vorhandene große Datensätze früherer Studien. So konnten sie auf über 20000 Bewertungen von mehr als 140 Ärzten zurückgreifen und die Diagnosegenauigkeit der einzelnen Ärzte berechnen. Mit diesen Informationen simulierten sie, unter welchen Bedingungen die mittels Regeln der kollektiven Intelligenz kombinierten Diagnosen treffsicherer sind als Einzeldiagnosen. Angewendet wurden dabei die Konfidenz- und die Mehrheitsregel. Während bei der Konfidenzregel pro Fall die Diagnose desjenigen Arztes gilt, der sich seiner Einschätzung am sichersten ist, gilt bei der Mehrheitsregel pro Fall diejenige Diagnose, welche am häufigsten von den Ärzten genannt wurde.

Nach einer Mitteilung des Max-PlanckInstituts für Bildungsforschung, Berlin 